sible. Le soleil d anlleur's, ne semblat pas encore éclaner la terre ; I ivalporation, les refroldissements nocturnes, toutes les conditions répulées nécessaires i la formation de la pluie paraissent alors mexistantes ? Le rent meme alors élant-il possible ?

Sans doute, durant les ípoques survantes, la diversité des '-limats s'est accuséc ; les rayons solaires attelgnament directement Ir sol; les pòles se précisarent : Cependant aucune époque géologrque ne prósente universollement de traces sensibles d'une circufation mtense des caux phuvales avant la fin de l'époque tertiarre. I a pluse, la vrane phue, par tusion de la nerge, existail-elle auparavant? Nous ne le pensons pas.

En effet, la pluic ctant, mar hypothèse et aussi d'après les fauts reconnus en dimnution progressive et constante, il est difficite d'admettre qu'cllo at tout d'abord débuté par de taibles chutes pour s'accroitre ensulte et dimmuer progressivement depuis lors. Nous pensons. tout au contraıre, que l'époque du maximum des précipitations aqueuses est précisément d’èpoque même de leur origine. Et nous f'assons remonter l'origme de la neige, et par conséquent de la phuie par vraies gouttes d'eau la l'exclusion de la plure fine possible, ì toute époque) lors de l'époque plérstocène ou au début du quaternarre. Les glaciers alor's se montrèrent sur loute la lcrre el les phénomènes d érosion et de circulatıon des caux devinrent, comme on be salt, vrament prodigieux à la surface du globe.

La conclusion de cette communcation est quil faut établir, par res chifres exacts et précis si, au moins en France, la dummulion des pluies est générale, quelle que soit la topographie et l'mportance du régime forestıer de la région considérée.

En second lieu, si, comme nous le croyons, les réglons bolsées subissent mons que toutes les autres l'assèchement dont se pla1gnent les pays dénudés, il serait à souhaiter que l'Association émette Ic vœu qu'une loi protège l'exustence des forêtis encore existantes et qu'une autre doi active le reboisement, principalement sur les hauteurs incultes ou en friche. Car il n'est guère de fléan plus grave, pour l'humanité entière, que celui d'un assèchement imiversel et il n'est pas trop des effortis de tout le genre humain pour se préserver d'une éventualité aussi redoutable.

\section{INSTRUCTIONS SUR LE MONTAGE DES INSTALLATIONS ÉLECTRIQUES}

Instrurluons redigées par les Associatıons françases de Propriétares lidpuruls à vapelur ayant un service ćlectrique (Amiens, Lyon, Marselle, Mulhouse-Nancy, par lissociation des Inrlustruels tu Nord de la France (Lille), el par l'Association normande pour prévenir les accidents.

(Suile el fin)

\section{L. - MONTIGE DES J.IGNLS}

\$3. - lrolecton. - a) Tous les conducteurs et appareils dinvent ilre mstallés de telle faģon qu'on puisse en tout temps Ins controler ou les remplacer

b) In leva mum d'enveloppes protectrices loutes les parties les lignes exposćes a des détériorations mécaniques (en partıculer aux (narconsées des planchens).

Ces enveloppes poumront ctre constutuées par des tubes et des moulures ou des carsisettes, grillages el filets.

() On devra velller altentivement ì ce que les fils et leurs supports ne soient jamais enduits de substances pouvant attaquer lisolant ou lo conducleur. Partout nil les fils et les supports se-

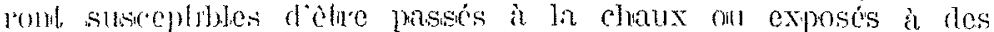
vapeur's coldosives, ils deviront stre, dies le montage, endurts dial moms doux rouches de pemlure latuée.

(d) Tes caljles sous plomb ne donvent jamais etre mis en confach mmodial aves des corps quil altaquent le plomb (le platie

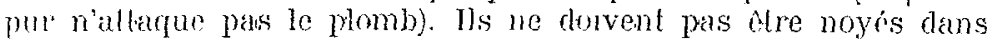
la macotinerie. Aux pomts de fixalton, l'envelopipe ile plomb no dul, difre ni écrasce ni entamée : l'emploi de clochets à tuyaux pour la fixation est done prohubé.

\$ 35. - Connexions. Epissures. - "1) Les comnexions des lignes avec les tablenux et les apparells ne doivent pas ctre réalisces par sondures ou ligalures, mais par serrage de vis assurant un rontact suffisaut el churable.

b) Jes conducteurs ayant plus de 25 millim. carrés de sec- tion et tous les cables devront être pourvus de pièces termmales appropr'tées ou d'un dispcrsitif équrvalent.

c) Les connexions des fils et câblès cntre cux ne peuvent chl'c ellecluées que par soudure ou un mode de jonction équivalenl.

(d) Les épissures ne dolvent avour à supporter aucun efforl de traction. Elles seront isolées avec soin et conformément à la nature de lisolation des conducteurs reliés.

e) Les connexions de cables sous plomb itanche (\$27) culle eux et à d'autnes conducteurs et appareils ne peuvent se lairc qu'i l'arde de manchons, boites de dérivation ou appareils analogues empêchant sûrement l'intrusion de l'humadité.

$\$ 36$. - Traversées des murs et plafonds. - a) Le passage des murs, cloisons et plafonds pourma s'eftectuer solt par une ouvelture de dimensions telles que l'écartement du conducteur el des paross reste celus prévu au paragraphe 38-e et $f$, soit en encastrant dans les murs des tuyaux isolants inallérables et résistants.

La dimension et la forme de ces tuyaux ainsi que leur sallie sur les panols seront appropriées à la tension du courant.

b) Quand la traversée se fera vers l'extérieur ou vers un local mouillé, on ne pourra employer, comme tube isolant, que la porcelaine, le verre ou des matières analogues et la disposition du tube devra empecher l'entrée et l'accumulation de l'eau, ce qui est réalısé en génénal en donnant aux extrémités la forme de prpes.

c) Pour les traversées, on doit, en règle générale, ne mettre qu'un fil par tube.

Pour la moyenne et la haute tension, l'observation de cetie règle est indispensable

\$37. - Lignes exlérieures et aériennes. - a) Les conducteurs dolvent avolr une résistance mécanique suffisante pour qu'il n'y ait aucun danger de rupture sous l'action des efforts qu'ils auront à supporler (Dimensions minima, voir \& 29).

b) Les supports doivent présenter toutes les garanties de soldité nécessaires. En particulıer, les supports en bois doivent elre prémunis contre laction de l'humidilé.

c) Pour les lignes extérieures non prolégées contre la pluie, on ne pourra employer que des isolateurs à cloches placés verticalement ou des dispositifs équivalents appropriés à la tension (1).

d) Les distances minima admisies entre conducteurs nus ou entre ceux-ci et toute partie du bâtıment seront déterminćes par la règlc suivante : $1 \mathrm{~cm}$. par 1.000 volts, plus $1 \mathrm{~cm}$. par mètre de portée avec un minimum de 10 cenimètres.

Pour l'cutróc dans les appareals, on devra mamtenur aulant que possible ces mimes ccartements des murs.

e) En dehors des vores publiques et quand il n'y a pas d'atutres règlements à appliquer, les conducteurs nus seront placés à une hauteur de 1 m. au moms au-dessus du sol

Pour la morienne et la haute tension, cette hauteur sera portóe ì 6 mètres.

Pour les conducteurs passant sur les torts ou le long des bitimonls, il faudra observer les règles suvantes:

Basse tension. - Mettre les conducteurs nus autant que possible hol's de portée.

Moyenne tension. - Isoler à 600 mógohms tous les colldnc. leurs qui ne pourront pas être mis surrement hous de portée (ha gaine extérieure devra rúsister aux mlemper'ies).

Haute tension. - Fviter le passage des conducteurs sur les toils et le long des batiments, ou les entourel de filels ou grillages pnotecteurs mis is la terre.

f) Lorsra'une ligne de télécommunication (sonnerie, lélóphone, etc.) esst posice sur les momes sumports que les lignes industrielles, clle doit toujours etre plache au-dessous de ces dernioress.

En outre, des prégatuons spéciales devroul dpre proses pour coarter tom danger on oas do contact entre les doux lignes (coupe-curemuls, lapts isolants, montage soigné). Dams ce (as, les

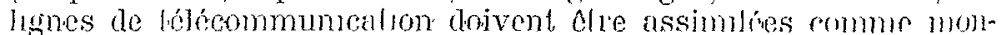
lage aux lignes industruelles qu clles suivent, tanl on lignes conrantes quai l'mtérieur des batiments, et les apparols aurfuels ches aboutissent doivent oftre protegós ol dispmesés do mamfinr it icalter tout danger (coupc-circuit, tapis isolant, elc.)

9) Pour la moyenne et la havte tension, on prendra on plus les précautions suivantes :

(1) Pour les lisnes de rontact, telles que celles employécs pour Ja traction, deux isolateurs simples placés en sípie dans l'apparesl to sus. pension scront considirés comme équivalents à un isolateur à double cloche. 
$1^{0}$ Les supporls accessibles dorvent ètre munts, sul lute hauteur de 50 centim. à partir de $2 \mathrm{~m}$. au-dlessus du sol, de dispositions spéciales pour empecher autant que possible d'atlenutre les conducleurs ;

$2^{\circ}$ Les supports mótalliques accessibles seront pourvus d une bonne communication avec le sol ;

$3^{\circ}$ Sur les apmus d'angles, on prendra les dispositumes nicessares pour relenrr les conducteur's au cas où lls vendraient i abandonner les isolateurs ;

1. Pour la traversée des voies publiques ef dans lous les cas où la chnte d'un conducteur serait susceptible de compromettre la sécurité de la circulation, il sera ólablı un disposiluf de protection au-dessous des conducteur's, à moms que ceux-c1 ne sorent rendus inoffensil's en cas de rupture. L'installation devra, en outre, se conformer aux prescriptions admminstratives,

$5^{\circ}$ Las disposutifs de protection des lignes aćlienues (cadres, filets) seront isolés ou mis à la terre de telle facon qu en cas de fonchonnement, aucun pont accessible du support ne puisse prendre par rapport it la terre whe différence de potentrel supérieure à 25 volts ;

$6^{\circ}$ Pour la haute tention, les haubans des supports derront être ssolés à une hauleur d'au moms $3 \mathrm{~m}$. au-dessus du sol ; seront igalement isolés, les haubans fixés ì des bitiments;

$\tau^{\circ}$ Les supports accessibles por leront linscription " "Dangereux, défense absolue de toucher aux fils, nìme tombés à terre ", ainsi que l'mdication de la tension;

$8^{\circ}$ Autant que possible, on évitera de poser sur les mèmes poleaux des lignes de basse, moyenne cl haute tension.

Quand la pose sur los menres supports ne pourra pas itre évitie, on devra placer la ligne a haute tension au-dessus de la ligne ¿ basse tension et un filet entre les deux. La distance entre les fils de ces deux lignes ne devra pas etne mférieure à 1 mètre.

$\$ 38$. - Lignes à l'intérieur des batments. - Conducteurs nus. - Les conducteurs nus ne peuvent être montés que sur isolateurs ì cloches ou sur poulies it nervures ct poulies-cloches appropriées à la tension.

Les distances mmima admises entre conducteurs nus sont les memes que celles indiquées pour les lignes extérıeures $\{\$ 37-d)$.

Jusqu'à 600 volts, lécartement des fils nus aux murs pourra être réduit à 5 centmm. pour des portées ne dépassant pas $1 \mathrm{~m}$. 50 . Pour l'entrée dans les apparells, on devra maintenur autant que possible ces mèmes écartements des murs. Pour les conducieurs reliant les accumulateurs au tableau, on peut admettre des distances plus faibles et des poulies de plus petites dimensions, tant que la tension de décharge ne dépassera pas 500 volts.

a) Basse tension. - L'emplor des conducteurs nus à l'mtéricur est limité aux salles de machines et d'accumulateurs, et aux locaux industriels incombustıbles ne contenant pas de malières mflammables, ì la condition de les protéger de manire ì les soustraire a tout contact accidentel.

Exceptionnellement, les conducteurs nus pourront ctre admis dans des locaux non incombustibles, mass ne contenant pas de matières faclemient inflammables :

$1^{\circ}$ Comme ligne de contact ;

$2^{\circ}$ Lorsqu'll y a dégagement de vapeurs corrosives, à condılion de les recouvirir d'un enduit qui les protège contre les corrosions

b) Moyenne tension. - Les conducleuns nus ne pourront itre cmployés que dans les salles de machines et d'accumulateurs el comme lignes de contact, it la condition d'être protégés confre tout contact accidentel wu d'ctre disposés de telle sorle fue lon no puisse les attendre (fu'en ćlant isolé du sol.

c) Ilaute tension. - L'omploi des conducteurs nus esi limité aux cabines de translormalcurs ef anx postes de distrubution. On pourra également tolérer leur passage à travers les salles de machines, it condition de les proléger.

d) Conducteurs isolés. - d) l'une manère générale, le montage des lignes sera fail de facon que les conducteurs ne puissent foucher, meme à l'entrée dans les appareils, les murs, parois et anthes objets conducteurs ou facilement mflammables.

c) Dans les locaux humides ot mounlés, la distance des conducteurs aux murs est déterminéc par les dimensions des isolateurs adnis, mais elle ne devra jamais êlre inféricure à 2 centimètres.

d) Prour la haute tension, la distance entre les conducteurs et les murs ou autres parties du balment sera de 1 centim. carré par 1.000 volls, avec mınımum de 2 cenlimètres.

11) Les conducteurs isolés doivent etre écartés les uns des auIres ; cependant il est permis, jusqu'd 150 volts, de torsader ou fle poser dans un meme tube des fils ayant an moins une isola-
Lon moyente ; jusquid 300 volls des hls dy ant au moms lune isolation forte (300) mégohns) et très forte (600 nrégohms), e! jusqu'i 600 rolts des fils ayant une isolation supermeure 1200 mégohms). Au-clessus de 600 volts, lemplor de fils lorsadés est interdit.

Les fils amsi torsades ne peuvent etre employes que dans les conditions ou les fils souples sont admis. Lor'sque, par sule de crroonstances particulieres, on sera obligé de l'éumr flus de trons fils en paquet, ces fils deviont avorr une isolation plus forte que celle normalement uécessaure, et, en outre, les fils de polalrités dilférentes devionl etre solgneusement sómarés.

h) Lorsque, aux crossements de conductems milre eux on arec d'autres pièces nuclalluues, il sera mupossible de mamtenar un écartement suffisant, Il faudra mterposer une isclation supplimentaure. Cefte isolation supplómentare devra ctre fixée sotgneusement.

v) Le long des murs, l'écartement des supports aulres que les cloches ne sera pas supérleur at $1 \mathrm{~m}$. 50. Le long des plafonds, leur écaltoment pourra ètre supéreur quand cela sela nicessamer pour adapter le monlage à la construction du plafond.

2) Cordons multiples et cordons souples. - Les fils souples multiples ne peuvent dre montés it demeure que dans des locaux parfaitement secs dans lesquels il n'y a ni production, ni accumulation de melanges explosifs, el à une distance de 3 millım. au moms des murs et des plafonds. Jians Jes appartements, on peul lokerer la fixation par altaches isolamles pour les fils somples, isolés ì 300 ou à 600 mégolims.

i) On dont éviter autant que possible d'employer des conducleurs multiples pour l'arrivéc anx mlerrupleurs el commutaleur's. llans les locaux où il se produl des poussieres ou des duvets liflammables sattachant aux fils, cet emplon ne peut etre toléré.

l) L'emplor de ligatures mélallicues ponr les conducteurs multiples est interdit.

m) Pour la jonclion des fils souples entre eux el avec d'autres conducteurs, ll est recommandable de laure usage de rosaces avec contacts vissés.

n) Les dérivalions de fils souples mobiles ne peuvent se faire que par l'interméduare de prises de courant ì fiche ou appareils cquivalents.

o) Lorsque les fils souples mobiles risquent de tremper dans l'eau (comme par exemple dans les brasseries, lemtureries, etc.), lis doivent etre entourés dun tuyau en caoulchouc formé herméliquement aux deux extrémités.

p) Pour la moyenne tension, on ne peut employer que des fils suuples a isolation forte recouverts dune game protectrice supplémentarre. Si ces cables ont une armature métallique, elle devra etre mise à la terre.

q) Pour la haute tension, l'emploi des conducteurs mobiles est interdit.

\$ 39. - Lignes souterraines. - a) Les conducteurs quels qu'lls soient doivent être protégés mécanıquement conlre les avaries que pourraient leur occasionner le tassement des teries, le con. tact des corps durs ou le choc des outlls en cas de foulle.

b) Les cables sous plomb avec armature en fer ou en acier pourront être posés directement dans le sol ; tous les autres dolvent être protégés par des canıveaux ou luyaux en cmnent, grès, fonte ou par des disposilifs équivalents.

c) La disposition des réseaux souterrains doul che lelle que les caux et les gaz ne puissent s'accunnler ni dans les tuyaux of caniveaux mi dans les boites de branchement ou de jonction.

Ces dernières doivent etro faciles ì vétufier.

d) Les jonclions des conducleurs souterrains avec les antres parlies du réseau doivent etrc déconnectables.

e) Pour la moyenne et la haute tension, les parties métallicnes des boîtes de bianchement ou de jonction devront obe mises ì la terre.

$\$$ 10. - Lignes el prises de terre. - a) Il est intertit rlemployer la terre seule comme partie du circuit.

b) Les lignes de terre doivent ctre en cuivre et leurs seclions doivent être proportionnées aux intensités de courant qui peuvent les traverser, avec un minimum de 7 millim. carrés (diamètre 3 millim.).

c) Toutes les jonctions des lignes de terre, sauf les racorrds avec les machines et apparcils, doivent etre soulées.

d) Les lignes de terre doivent ctre mises a labri des deterion'ations mécaniques el chimiques.

c) Les prises de terre seront constituées :

10 Par des plarues, grillages nu antres conductomrs noyés dams 
des terrains humndes ol de dunensions sullisantes pour résister à laclion destructive du sol;

$20 \mathrm{Par}^{2}$ des condutes d'ean soulerrames étenducs ou autres masses mélalliques en cortact avec le sol ; ces dernières ne pour. ront d̀tre cmployées seules que pour les lerres deslmécs unquement a la prolection des persomnes (i l'exclusion des parafondres).

d) La risistance de la prise de terre dout otre aussı faible que possible, ne pas dépasser 20 ohms et, dans tous les cas, satısfurc aux condituons du paragraphe $1-g$.

Il $y$ aura lieu de soigner tout particulièrement la connexion onle la ligne el la prise de terre, surtout en ce qui concerne sa bonne conservation.

1) Il est trìs recommandé de mettre a la terre le fil neulre des liseaux à courant contmu à trois fils.

\$11. - Isolement de l'inslallation. - a) Toule partie d'une ins. lallawon pouvant etre séparée de l'ensemble par la manouvre A'un inleprupleur ou lenlevement d'un fusible devra présenter. lant par rapport a la terre qu'entre deux condueteurs de polarlés ou de pliases dillérentes, une rusistance d'isolement exprimée en ohms, au moins égale à $23000 \frac{E}{l}$, avec un minimum exıgıle de 5 millions d'ohms.

Dans colle formule, $E$ représente la tenston de régume en volts ct $l$ le couranl normal en ampères chrculant dans la partie constriric un seul récepteur ne pourra jamass compter pour ilus de 20 ampères.

b) D'ou' les parties d'une installation qui se trouvent dans des locanx moullés (brasseries, temtureryes, etc), lisolement exiglble sera au mons le disıème de celus mdrqué par la formule précédente.

c) Lorsqu'il s'agit dinstallations neuves, ll faut mesurer non seulement l'isolement des conducteurs par rapport à la terl e,inas aussi l'isolement entre eux des conducteurs de polentiel dufférent ; la mesure doit se farre, l'apparellage et les fusibles mis en place, les interrupteurs ćtant fermés. Dans ces conditions, les risistances disolement dowvent satisfaire ì la formule cr-dessus.

(l) Les lignes aćriennes devront prósenter me résistance disolement, exprimée en ohms au moms égale ì $5.000 \mathrm{E}$, avec un maximum exigıble de 10 mégohms par kilomètre de lıgne simple.

e) Toutes les mesures disolement donvent être effectuées de próférence it la tension de marche, maus au moins ì 100 volts.

f) IIaule tcnsion. - Toute installalion a haute tenswon doit ctre munke, à la statron génératrice, d'un appareil permeltant de vérłficr lisolement de linstallation en marrche.

\$ 41. - Affiches - a) Dans toutes les installations indistruelles de moyenne ct haute tension, ainst que dans les locaux industriels très mounlés, on mettra aux endroils appropriés des tableaux en caractères bien visibles, avertissant qu'll est dangercux de toucher aux conducteurs et apparels électriques, même avec des gants en caroutchouc on des outils it manche isolant

b) De mème on affichera des mistructuons sur le mode de tra!tement des personnes foudroyées par le courant éleclrique.

\section{TRANSPORT D'ÉNERGIE A 100000 VOLTS dans l'Ontario}

La IIydro-elec/ric Pourer Commission de ia provinco d'Ontario, au Canada, a commencé les travaux d'installation d'une distribution d'éneruie d'une longueur d'environ $500 \mathrm{~km}$., sous la tension utilisable de 100.000 volts $\left(^{*}\right)$

L'énergie sera fournie pal' l'usine du Niagara de l'Ontario Pover $C^{\circ}$, ou la tension sera élevée de 12.000 a 110.000 volts. La puissance actuelle de l'usine, qui est de 40.000 chevaux, sera portée ultérieurement à 55.000 chevaux. Le courant est triphasé à la fréquence 25.

Ainsi que le montre le plan général (fig. 1) nor'd-est, la ligne partant des chutes se dirige d'abord vers le nord, et atteint Dundas à $80 \mathrm{~km}$.; de là, une dérivation de $65 \mathrm{~km}$. dessert à l'est Toronto, tandis qu'à l'opposé, deux lignes séparées

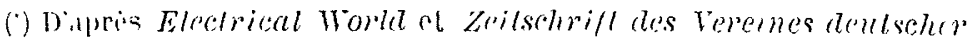
lngenieure. relient Dundas à London; l'une de ces lignes, la plus au nord, a une longueur de $200 \mathrm{~km}$., l'autre a 120 kilomètres.

London sera relié par une ligne de $24 \mathrm{~km}$ à Saint-Thomas, ou s'arrêtent les travaux en cours; mais on comple prolonger la ligne jusqu'à Windsor, à $160 \mathrm{~km}$ plus loin.

Ainsi que l'usage s'cn est établi en Amérique, pour les lignes a haute tension, on utilise des pylones, représentés fig. 2, qui sont constitués pal des fers comières, et qui sont placés à une grande distance los uns des autres, de manièro à avoir un nombre faible de points d'appui, ce qui assure un meilleur isolement. Les portées en ligne droite seront de $170 \mathrm{~m}$. euviron, et en courbe de $40 \mathrm{~m}$.; à la traverséc rlo la rivière Ilumber, la portce sera de $400 \mathrm{~m}$. La hauteur dos

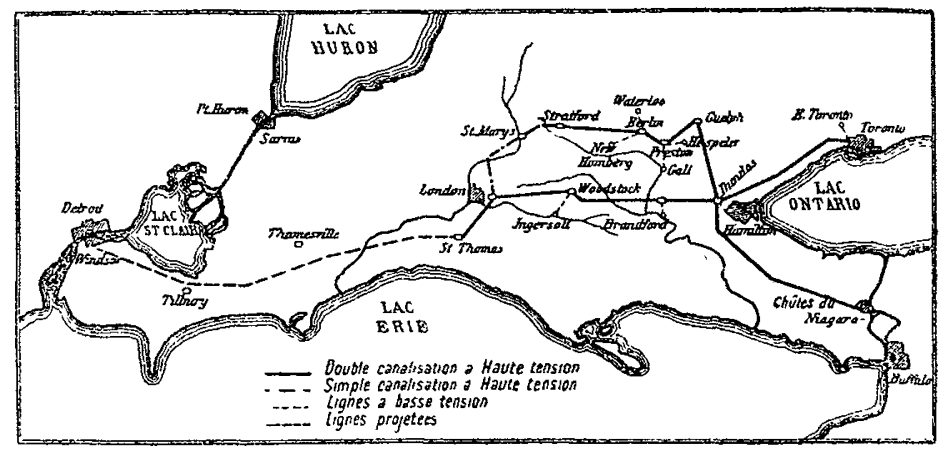

Fia. 1. - Plan général du réseau de dislribution.

poteaux ordinaires est de $20 \mathrm{~m}$. et le point le plus luas des conducteurs a $7 \mathrm{~m}$. au-dessus du sol; à la traversêo du canal de Welland, les fils seront à $45 \mathrm{~m}$. au-dessus du niveau de l'eau, afin de laisser libre passage aux bateaux.

Les isolateurs sont du type suspendu, et à unités multiples, décrit par M. Hewlett dans le numéro de juillet 1907 des Proceedings of the American Institute of Elec/rical Engineers; chaque unité est constituée par un disque ell porcelaine, les diverses unités sont reliées entre elles par
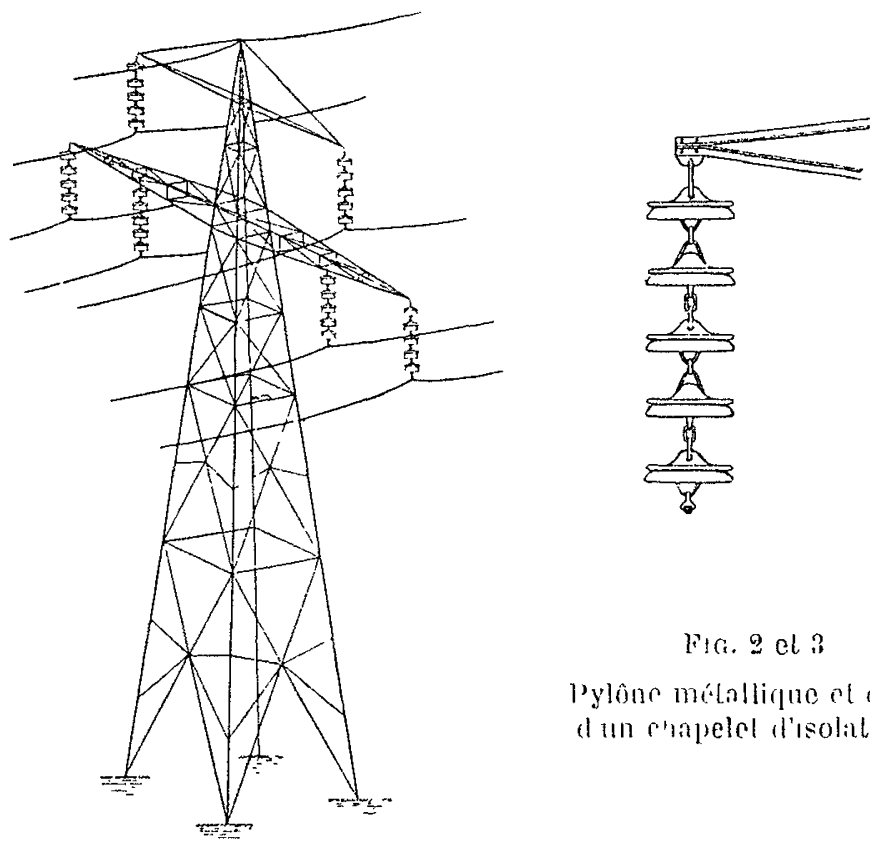

Fict. 2 el?

pylône mátollirgue el drítal d won phapelel d'isolialouri.

des câbles en acier. Dans lo type avec disquo de $25 \% \mathrm{~mm}$ de diamètre, un are ne se forme que pour une tension de 6 i 000 volts.

Dans les portions qui sont en ligne droite, quand lo conducteur' n'a pas besoin d'être arrêté, celui-ci est simplemont suspendu à la partie inférieure de l'isolateur qui est altaché aux bras transversaux des poteaux, de sorte que cet isolateur pend verticalement. Aux points où un conducteur doit être arrêté, c'est-à-dire tous los cinq ou six poteaux environ on ligne droite, ainsi que dans les courbes, l'cxtrémité du conductenr est fixé à l’isolateur, qui esl alors 